\title{
Open
}

\section{Double-stranded RNA induces molecular and inflammatory signatures that are directly relevant to COPD}

\author{
P Harris ${ }^{1,5}$, S Sridhar ${ }^{2,5}$, R Peng ${ }^{1}$, JE Phillips ${ }^{1}$, RG Cohn $^{1}$, L Burns ${ }^{1}$, J Woods ${ }^{1}$, M Ramanujam ${ }^{1}$, \\ M Loubeau ${ }^{1}$, G Tyagi ${ }^{3}$, J Allard ${ }^{2}$, M Burczynski ${ }^{2}$, P Ravindran ${ }^{2}$, D Cheng ${ }^{2}$, H Bitter ${ }^{2}$, JS Fine ${ }^{1}$, \\ CMT Bauer $^{1}$ and CS Stevenson ${ }^{1,4}$
}

Polyinosinic:polycytidylic acid (poly I:C) is a synthetic analogue of double-stranded (ds)RNA, a molecular pattern associated with viral infections, that is used to exacerbate inflammation in lung injury models. Despite its frequent use, there are no detailed studies of the responses elicited by a single topical administration of poly I:C to the lungs of mice. Our data provides the first demonstration that the molecular responses in the airways induced by poly I:C correlate to those observed in the lungs of chronic obstructive pulmonary disease (COPD) patients. These expression data also revealed three distinct phases of response to poly $\mathrm{I}: \mathrm{C}$, consistent with the changing inflammatory cell infiltrate in the airways. Poly I:C induced increased numbers of neutrophils and natural killer cells in the airways, which were blocked by CXCR2 and CCR5 antagonists, respectively. Using gene set variation analysis on representative clinical data sets, gene sets defined by poly I:C-induced differentially expressed genes were enriched in the molecular profiles of COPD but not idiopathic pulmonary fibrosis patients. Collectively, these data represent a new approach for validating the clinical relevance of preclinical animal models and demonstrate that a dual CXCR2/CCR5 antagonist may be an effective treatment for COPD patients.

\section{INTRODUCTION}

Respiratory viral infections lead to significant deteriorations in the health of individuals with underlying lung pathologies. These infections can trigger acute exacerbations, episodic events that in many cases lead to hospitalization, worsened pulmonary function, and are the major causes of morbidity and mortality. The vast majority of the infections associated with acute exacerbations of chronic obstructive pulmonary disease (COPD) are due to rhinovirus and influenza. ${ }^{1-3}$ Presently, it is not understood why or how these infections trigger acute exacerbations and as such there are no treatments that can effectively prevent or attenuate the damage caused by these events in patients.

The lack of effective therapies for treating exacerbations is due, in part, to a paucity of preclinical in vivo models that are known to reflect the changes (and mechanisms) associated with exacerbations. Polyinosinic:polycytidylic acid (poly I: C) is a synthetic analogue of double-stranded (ds)RNA used in preclinical models to mimic the replication intermediates present in cells infected with RNA viruses. ${ }^{4}$ It is well understood that dsRNA and its synthetic mimetic, poly I:C, can activate pattern-recognition receptors (i.e., Toll-like receptor (TLR) 3 and RNA helicases). ${ }^{5-7}$ Although poly I:C has been used to exacerbate the immune response in models of cigarette smoke-induced airway inflammation, ${ }^{8}$ no studies have comprehensively described the responses induced by a single administration of this dsRNA synthetic analogue to the lungs. Understanding these mechanisms is required in order to determine how dsRNA interacts with other environmental challenges such as cigarette smoke to impair pulmonary function.

\footnotetext{
${ }^{1}$ DTA Inflammation, Hoffmann-La Roche Inc., pRED, Pharma Research and Early Development, Nutley, New Jersey, USA. ${ }^{2}$ Translational Research Sciences, HoffmannLa Roche Inc., pRED, Pharma Research and Early Development, Nutley, New Jersey, USA. ${ }^{3}$ Non-Clinical Safety, Hoffmann-La Roche Inc., pRED, Pharma Research and Early Development, Nutley, New Jersey, USA. ${ }^{4}$ Respiratory Pharmacology Group, Pharmacology and Toxicology Section, Centre for Respiratory Infections, National Heart and Lung Institute, Imperial College London, London, UK. ${ }^{5}$ These authors contributed equally to this work. Correspondence: CS Stevenson (Christopher.stevenson@roche.com) 
Another shortcoming of these preclinical models is the lack of an unbiased approach for determining whether the molecular changes observed in the model are directly relevant to changes which occur in the clinic. Matching the models to the correct patient populations, in addition to determining what mechanisms are translational and when they occur, will be critical for successfully predicting the efficacy of drug candidates using these systems.

Thus, our aim was to provide a systematic characterization of the molecular, cellular, and physiological changes elicited by a single intranasal (IN) administration of poly I:C to mice. These data allowed us to define unique molecular signatures associated with the immune response to dsRNA. Further, we validated these mechanisms using pharmacological tools to block the inflammatory cell infiltrate induced by poly I:C. To determine whether these molecular changes were translated in a clinical setting, we used a novel method for assessing the enrichment of these gene sets in individual patient samples called gene set variation analysis (GSVA). ${ }^{9}$ This approach evaluates the enrichment of specific gene sets within the individual samples rather than within the treatment groups, which is a distinct advantage over gene set-enrichment analysis (GSEA). As such, this allows investigators to identify select gene sets that define and differentiate individuals within populations (i.e., disease subpopulations). Using this method, we show for the first time that poly I:C-induced signatures were enriched in clinical data sets generated from the lungs of COPD patients, but not in patients with other smoking-related diseases (i.e., idiopathic pulmonary fibrosis (IPF)) relative to their respective controls. Together, these data reveal a comprehensive dissection of the mechanisms driving dsRNA-mediated inflammation and suggest that chemokine receptor antagonists that block neutrophil and natural killer (NK) cell migration (i.e., CXCR2 and CCR5, respectively) may provide a therapeutic benefit to patients with COPD but not IPF.

\section{RESULTS \\ Poly I:C induces airway inflammation and airway hyper-responsiveness (AHR)}

To identify a dose of poly I:C that elicited a sub-maximal inflammatory response, we initially performed a dose-response study. Poly I:C administered IN to mice dose-dependently increased bronchoalveolar lavage fluid (BALF) neutrophil and lymphocyte numbers. A dose of $30 \mu \mathrm{g}$ was chosen for all subsequent studies (see Supplementary Figure S1 online). The decreased neutrophil numbers at the highest dose were attributable to poor delivery of poly I:C due to the increased viscosity of the solution at this high concentration (see Supplementary Figure S1B online). BALF neutrophils increased starting at $6 \mathrm{~h}$ post-poly I:C administration and peaked with a 14 -fold increase at $48 \mathrm{~h}$ relative to the time-matched controls (Figure 1a). BALF lymphocyte numbers increased from 24 to $168 \mathrm{~h}$. Infiltrates of lymphocytes, neutrophils, and some macrophages localized to the peribronchiolar and perivascular
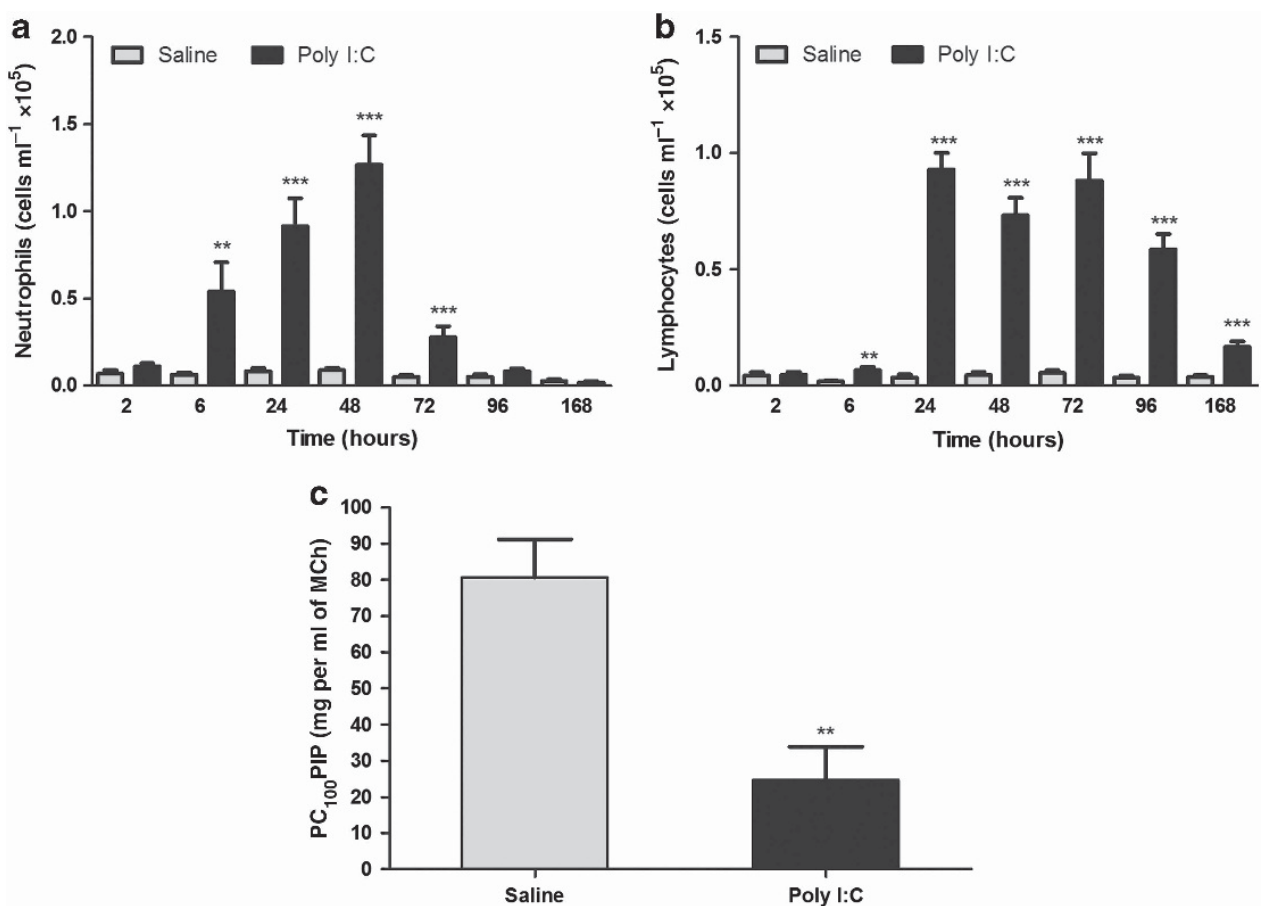

Figure 1 Neutrophils and lymphocytes infiltrate the airways in a time dependent manner in response to polyinosinic:polycytidylic acid (poly I:C). Inflammatory cell numbers increased in the bronchoalveolar lavage fluid (BALF) from $2 \mathrm{~h}$ through $168 \mathrm{~h}$ post-poly I:C instillation. Differential cell counts revealed that (a) neutrophils and (b) lymphocytes in the BALF were the predominant leukocyte populations that were elevated. (c) These changes were associated with airways hyper-responsive to cholinergic agents indicated by the reduced $\mathrm{PC}{ }_{100} \mathrm{PIP}$ (provocative concentration producing a $100 \%$ increase in pulmonary insufflation pressure) values. Data are expressed as mean \pm s.e.m. of $n=10-12$ mice. Significance (relative to the time-matched control at each time point) was determined using a Student's $t$-test and is denoted as follows: ${ }^{*} P<0.05 ;{ }^{* \star} P<0.01$; and ${ }^{* * *} P<0.001$. MCh, methacholine 
a

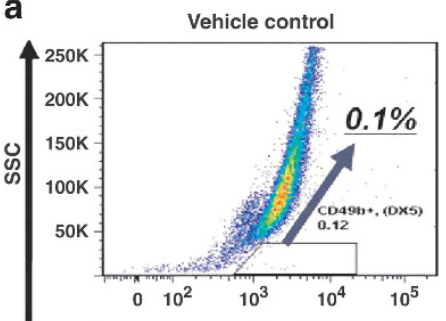

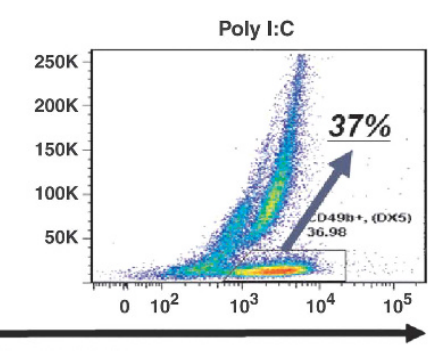

CD49b (DX5)

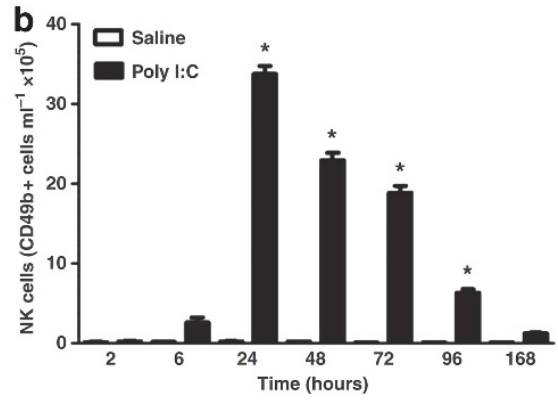

Figure 2 Natural killer (NK) cells are the primary lymphocyte population infiltrating the airways of polyinosinic:polycytidylic acid (poly I:C)-treated mice. (a) NK cells were determined by flow cytometric analysis by gating on the lymphocytes (low side scatter (SSC) gate) and CD49b + cells within the CD45+ cell population. (b) Total numbers were determined by multiplying the percentage of low SSC, CD49b+ cells, and the total numbers of cells recovered in the bronchoalveolar lavage fluid using an automated cell counter. Data are expressed as mean \pm s.e.m. of $n=10-12$. Significance (relative to the time-matched control at each time point) was determined using a Student's $t$-test and is denoted as ${ }^{*} P<0.05$.

regions of the lungs (see Supplementary Figure S2A,B online). The endothelial layer of many larger blood vessels was also partially lined by inflammatory cells. To a lesser extent, there were also alveolar and interstitial infiltrates (see Supplementary Figure S2C online). No goblet cell metaplasia was observed in sections stained using the alcian blue and periodic acid-Schiff method.

Airway responses to increasing doses of methacholine were measured non-invasively using whole-body plethysmography at 6,24 , and $48 \mathrm{~h}$ post-poly I:C administration. AHR was only observed at $24 \mathrm{~h}$ as determined by Penh area under the curve (see Supplementary Figure S3 online). Poly I:C-induced AHR was confirmed by measuring pulmonary insufflation pressures (PIPs) using invasive lung function techniques. AHR was determined by a drop in the concentration of methacholine that caused a $100 \%$ increase in PIP values from $80.8 \pm 10.4 \mathrm{mg} \mathrm{ml}^{-1}$ in the control mice to $24.7 \pm 9.3 \mathrm{mg} \mathrm{ml}^{-1}$ in the poly I:C-treated mice $(P<0.01$; Figure 1c).

\section{Inflammatory cell populations increased by poly I:C administration}

Flow cytometric analysis revealed that the lymphocytes in the BALF were predominantly CD49b + NK cells (Figure 2a,b). The percentage of CD $49 \mathrm{~b}+$ cells that were also CD3 + was $3 \%$ at $24 \mathrm{~h}$ and $7 \%$ at $96 \mathrm{~h}$ post-poly I:C administration, which suggests that the vast majority of the $\mathrm{CD} 49 \mathrm{~b}+$ population were conventional NK cells. Additionally, NK cells peaked at $48 \mathrm{~h}$, whereas CD3 + cells (Figure 3a) peaked slightly later at $72 \mathrm{~h}$ post-poly I:C instillation. In the CD3 + T-cell population, there was an increased proportion of CD8 + T cells compared with time-matched controls (Figure 3b), which was still evident at $168 \mathrm{~h}$, whereas the percentage of CD $4+\mathrm{T}$ cells was decreased (Figure $3 \mathrm{c}$ ). In the lung tissue, the presence of specific cell types was also assessed using GSEA based on published gene sets described for leukocyte populations in the blood. ${ }^{10}$ The molecular signatures for activated NK cells, monocytes, and dendritic cells all showed significant upregulation in the lung tissue from poly I:C vs. saline-treated mice $(P<0.05$, false discovery rate $(\mathrm{FDR})<0.05)$ between 6 and $48 \mathrm{~h}$ post-treatment. There was also a modest upregulation of these signatures at both the early $(2 \mathrm{~h})$ and later (72-96h) time points $(P<0.05$, FDR $<0.25$, see Supplementary Figure S4A online).

\section{Temporal molecular changes induced by poly I:C in the lung tissue}

Consistent with the changes in inflammatory cell infiltrates, microarray analysis revealed a tri-phasic pattern of differentially expressed genes (DEGs). Hierarchical clustering analysis of 1379 DEGs across all time points revealed four distinct clusters that consisted of mice from the control group, the 2-6h groups (early-phase), the 24-48 h groups (mid-phase), and the 72-96h groups (late-phase; Figure 4a). The mice from the $168 \mathrm{~h}$ group clustered among the controls, suggesting that the response to poly I:C had resolved.

The majority of the expression changes (both up- and downregulated genes) occurred at 6 and $24 \mathrm{~h}$. Gene ontology analysis indicated that immune response genes were upregulated at $2 \mathrm{~h}$, TLR pathways were upregulated at 6 and $24 \mathrm{~h}$, and cell cycle processes were increased at $72-96 \mathrm{~h}$. There were far fewer downregulated genes across time points, with ontology analysis revealing cell adhesion and musclespecific genes being downregulated at $24-48 \mathrm{~h}$. A separate literature-derived TLR3 signature consisting of genes-altered downstream of TLR3 was also shown to be generally upregulated in poly I:C-treated mice from 6 to $48 \mathrm{~h}$ post-treatment (Figure 4b). Interestingly, a subset of this gene set was more highly expressed in the saline-treated animals at later time points. Modular analysis of gene sets previously shown to be expressed in the blood of various disease cohorts ${ }^{11}$ was also performed, revealing upregulation of type I interferon genes from 2 to $96 \mathrm{~h}$. NK cell, monocyte, and dendritic cell modules were also upregulated, further validating the previously described GSEA results using a separate set of blood cell markers (see Supplementary Figure S4B online). Given the gene signatures used in both the GSEA and inflammatory module analysis were defined using purified cell populations from human whole blood, ${ }^{10,11}$ further validation may still be required to rule out the possible contributions of 

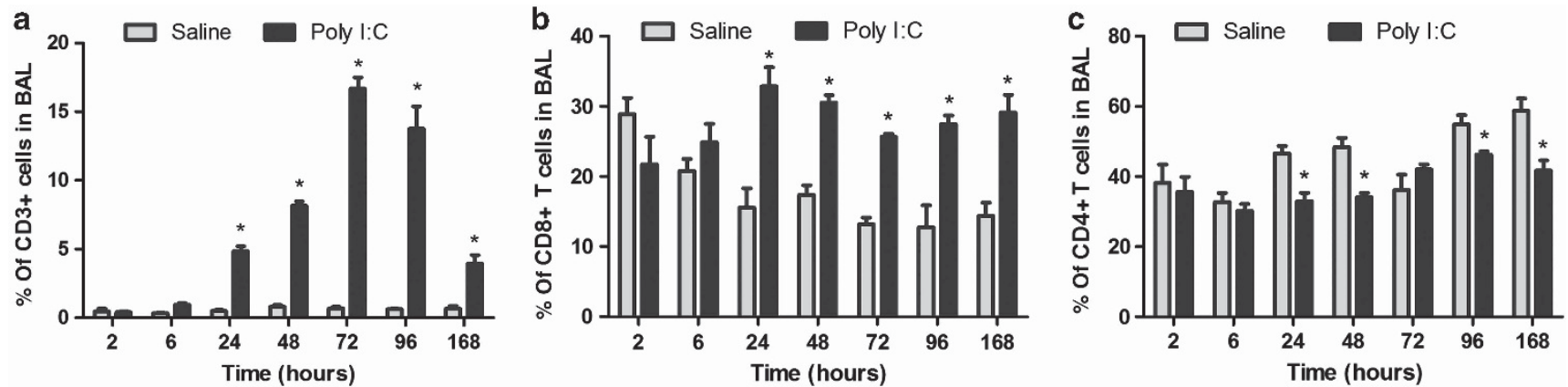

Figure 3 The percentage of CD8 + T cells are increased in the bronchoalveolar lavage (BAL) fluid of polyinosinic:polycytidylic acid (poly I:C)-treated mice. For the analysis of the T-cell subsets, from the total BAL cells, CD3 + T cells were gated on and are presented here as a percentage of (a) the CD45 + population. Percentages of (b) CD8 + and (c) CD4 + T cells are presented as percentages of CD3+ population from the CD45+ gate. Data are expressed as mean \pm s.e.m. of $n=10-12$ mice. Significance (relative to the time-matched control at each time point) was determined using a Student's $t$-test and is denoted as ${ }^{*} P<0.05$.
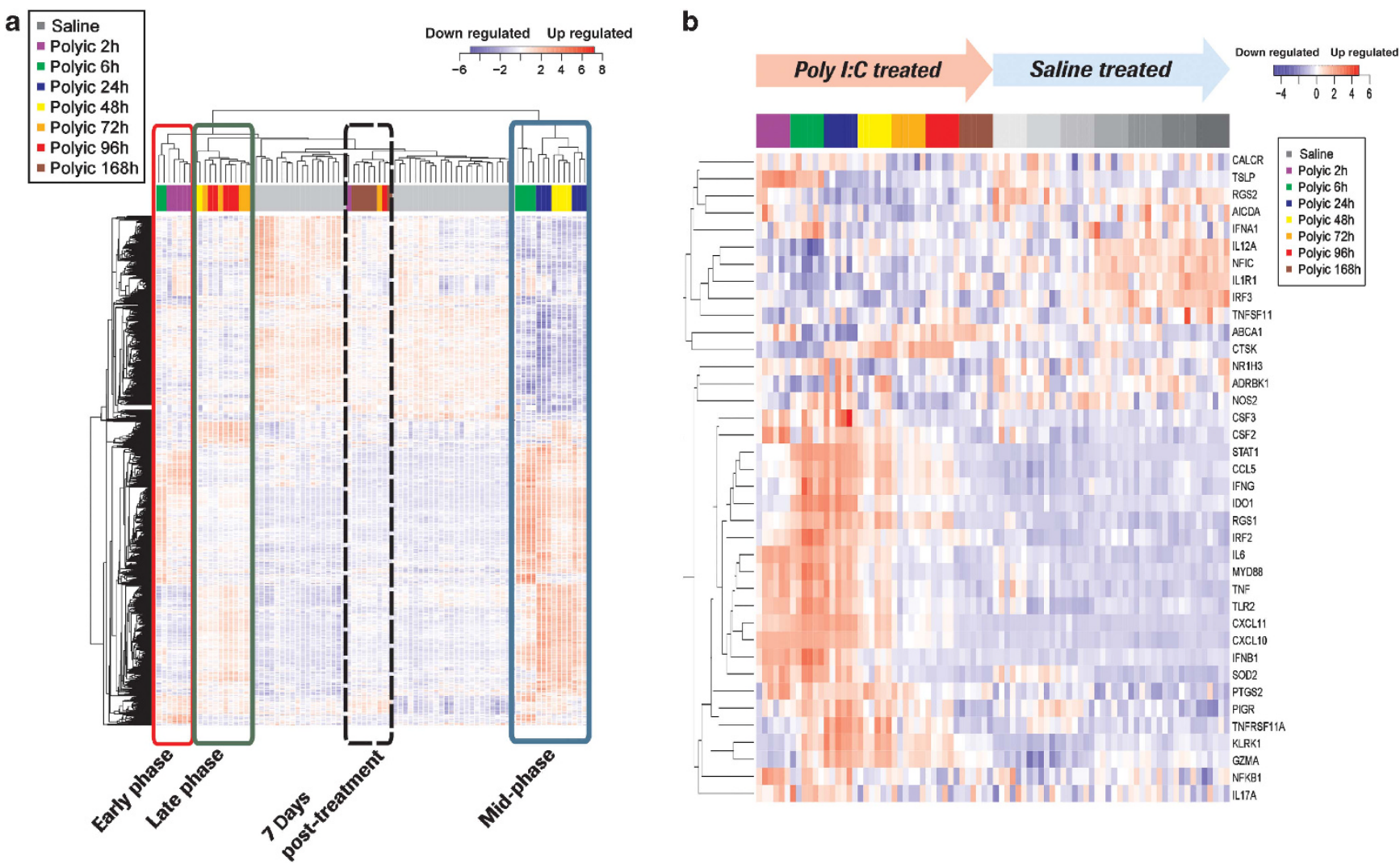

Figure 4 Hierarchical clustering analysis of polyinosinic:polycytidylic acid (poly I:C) response genes reveals tri-phasic response. Columns represent individual mice, rows represent genes. Upregulated genes are shown in shades of red, while downregulated genes are shaded blue. Poly I:C-treated mice are color coded by time point, saline-treated samples are colored in grey. Three distinct clusters of poly I:C-treated samples appear based on clustering analysis, with mid-phase (24-48h), early phase (2-6h), and late phase (72-96h) samples forming separate groups (a). Samples from the 7-day time point cluster with saline-treated samples. (b) Supervised clustering analysis of a custom-derived set of Toll-like receptor 3 (TLR3)-related genes indicate that this gene set is upregulated in poly I:C-treated mice during early and mid-phase. Columns represent samples, which are ordered and colored by treatment and time point. Rows represent TLR3 genes. Genes are clustered based on similar patterns of expression across treatment and time points.

non-leukocyte populations to the enrichment of these signatures in tissues other than blood. That said, these data do appear to be consistent with changes in cellular infiltrates that one would expect in this model.
Inflammatory mediators increased in BALF following poly I:C administration

A clear tri-phasic pattern in BALF mediator levels corroborated the changes observed at the molecular and cellular levels. In the 

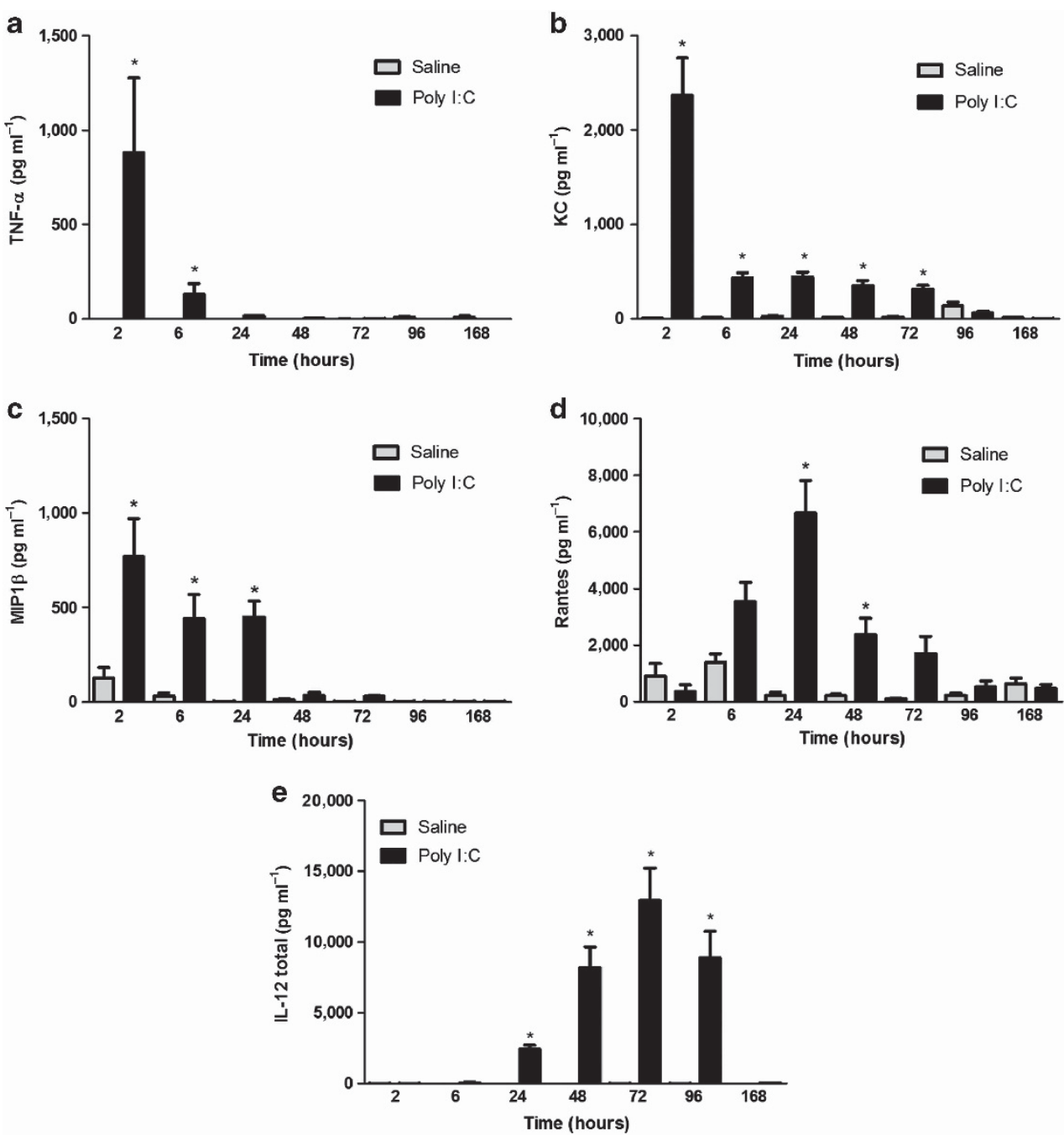

Figure 5 Polyinosinic:polycytidylic acid (poly l:C) induces cytokine and chemokine secretion in the bronchoalveolar lavage fluid (BALF). The BALF levels of (a) tumor necrosis factor- $\alpha$ (TNF- $\alpha$ ), (b) keratinocyte chemoattractant (KC), (c) macrophage inflammatory protein-1 $\beta$ (MIP-1 $\beta$ ), (d) RANTES (regulated on activation, normal T cell expressed and secreted), and (e) total interleukin (IL)-12 were measured by MSD. The following cytokines had no measurable change: IFN- $\gamma$ (interferon- $\gamma$ ), IL-1, IL-2, IL-4, IL-5, GM-CSF (granulocyte macrophages colony-stimulating factor), IL-13, and IL-17 (data not shown). Data are expressed as mean \pm s.e.m. of $n=11-12$ mice. Significance (relative to the time-matched control at each time point) was determined using a Student's $t$-test and is denoted as ${ }^{*} P<0.05$.

acute phase ( $2 \mathrm{~h}$ post-poly I:C), peak levels of keratinocyte chemoattractant $(\mathrm{KC})\left(2366 \pm 397 \mathrm{pg} \mathrm{ml}^{-1}\right)$, macrophage inflammatory protein $-1 \beta($ MIP- $1 \beta)\left(769 \pm 201 \mathrm{pg} \mathrm{ml}^{-1}\right)$, and tumor necrosis factor- $\alpha$ (TNF- $\alpha)\left(881 \pm 395 \mathrm{pg} \mathrm{ml}^{-1}\right)$ were observed in poly I:C-treated mice, whereas these mediators were below the limit of quantitation in time-matched controls (Figure $5 \mathbf{a}-\mathbf{c})$. In the mid-phase of the response $(6-24 \mathrm{~h})$, regulated on activation, normal $\mathrm{T}$ cell expressed and secreted (RANTES) peaked at $24 \mathrm{~h}$ (30-fold greater than controls). During the late phase, interleukin (IL)-12 peaked at $72 \mathrm{~h}\left(12930 \pm 2299 \mathrm{pg} \mathrm{ml}^{-1}\right.$, controls were below the limit of quantitation; Figure 5d,e). These data were consistent with message levels of these same cytokines and chemokines that were assessed by microarray analysis of whole-lung tissue RNA (see Supplementary Figure S5 online).

\section{Effects of a steroid and chemokine antagonists on} poly I:C-induced airway inflammation

To determine the mechanisms driving poly I:C-induced inflammatory cell infiltrates, we evaluated the effects of a broad acting anti-inflammatory (the steroid, budesonide), a CXCR2, and a CCR5 antagonist. Orally administered budesonide had no effect on BALF neutrophil, inflammatory protein-10, or KC levels, and on AHR (Figure 6a-g). Treatment with steroid did result in a dose-dependent decrease in BALF lymphocyte numbers $\left(83 \pm 6 \%\right.$ at $10 \mathrm{mg} \mathrm{kg}^{-1}, P<0.001$, Figure 6a). This inhibition was consistent with reduced NK cell numbers and reduced expression of the CCR5 ligand, MIP- $1 \beta(74 \pm 5 \%$; Figure 6c,e, respectively). Given that BALF KC levels preceded the neutrophil infiltrate and steroids did not affect BALF KC levels or neutrophil numbers, we tested the CXCR2 antagonist, 

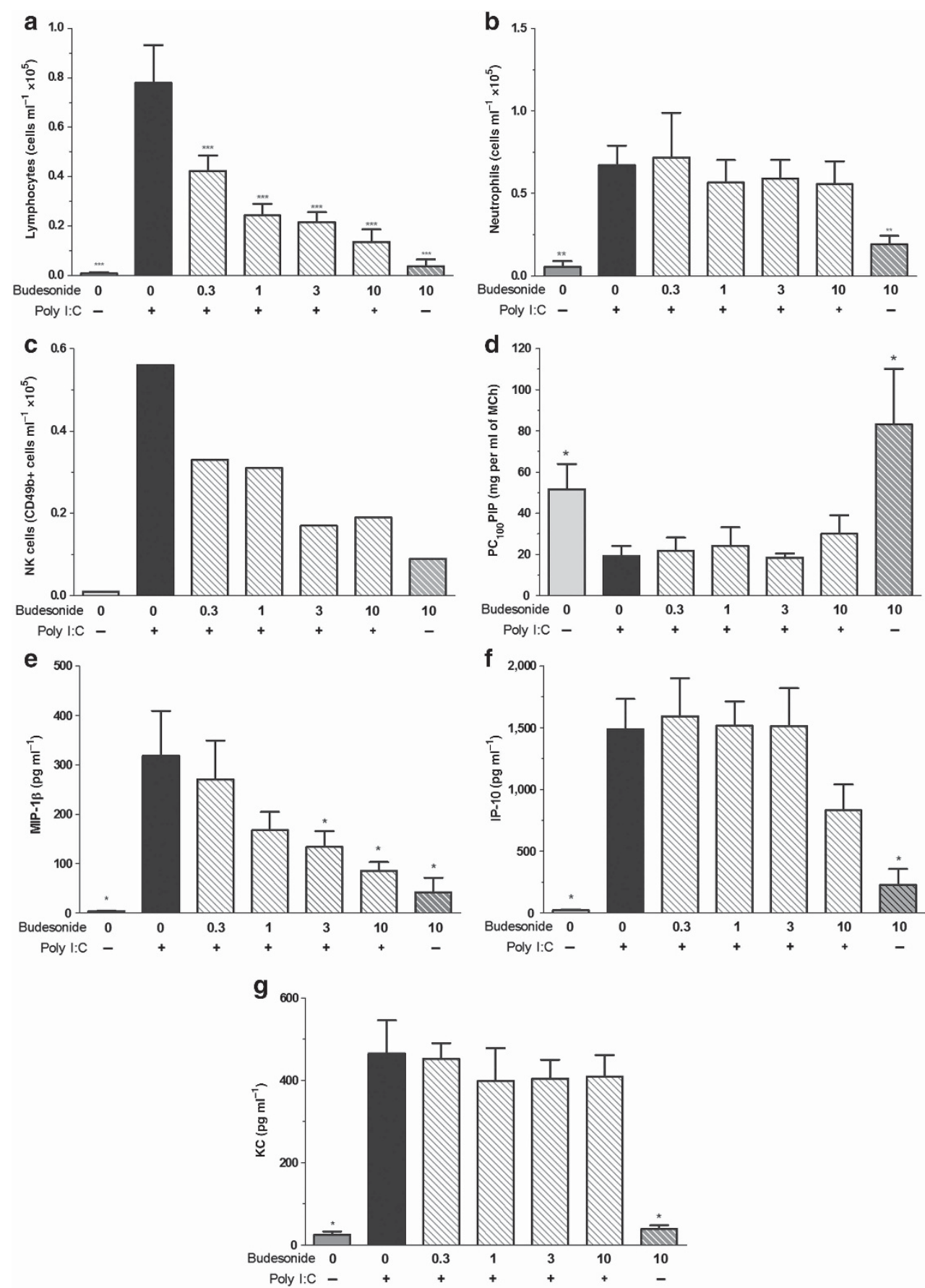

Figure 6 Effects of a glucocorticoid on polyinosinic:polycytidylic acid (poly l:C)-induced airway inflammation. Budesonide, administered $1 \mathrm{~h}$ before poly I:C instillation, dose-dependently reduced (a) bronchoalveolar lavage fluid (BALF) lymphocyte infiltrate but had no effect on (b) BALF neutrophils or (d) airway hyper-responsiveness. Neutrophil and lymphocyte numbers were determined by performing total and differential cell counts. The lymphocyte inhibition correlated with (c) natural killer (NK) cell decreases as measured by flow cytometric analysis of pooled samples $(n=8$ mice). Budesonide also reduced BALF levels of (e) macrophage inflammatory protein-1 $\beta$ (MIP-1 $1 \beta$ ) but had no effect on (f) inflammatory protein (IP)-10 or (g) keratinocyte chemoattractant $(\mathrm{KC})$ induced by poly I:C administration. Data expressed as mean \pm s.e.m. of $n=8-10$ mice. Significance (relative to the poly I:C vehicle control) was determined using a one-way analysis of variance and is denoted as follows: ${ }^{\star} P<0.05,{ }^{\star \star} P<0.01$; and ${ }^{\star \star \star} P<0.001$. MCh, methacholine; $\mathrm{PC}_{100} \mathrm{PIP}$, provocative concentration producing a $100 \%$ increase in pulmonary insufflation pressure.

SCH563705. ${ }^{12}$ SCH563705 inhibited the induction of neutrophils by $76 \pm 9 \%(P<0.05$, Figure $7 \mathbf{b})$ but had no effect on lymphocytes or AHR (Figure 7a,d, respectively).

We next assessed the CCR5 antagonist, TAK- $779,{ }^{13}$ to determine its effect on the lymphocytic infiltrate. The expression of CCR5 ligands in BALF (Figure $\mathbf{5 c}, \mathbf{d}$ ) preceded the NK cell infiltrate, which in addition to the data from the steroid study suggested that it had a role in NK cell chemotaxis. TAK-779 dose-dependently inhibited the total number of cells recovered in the lavage fluid by up to $66 \pm 13 \%$ (data not shown), 

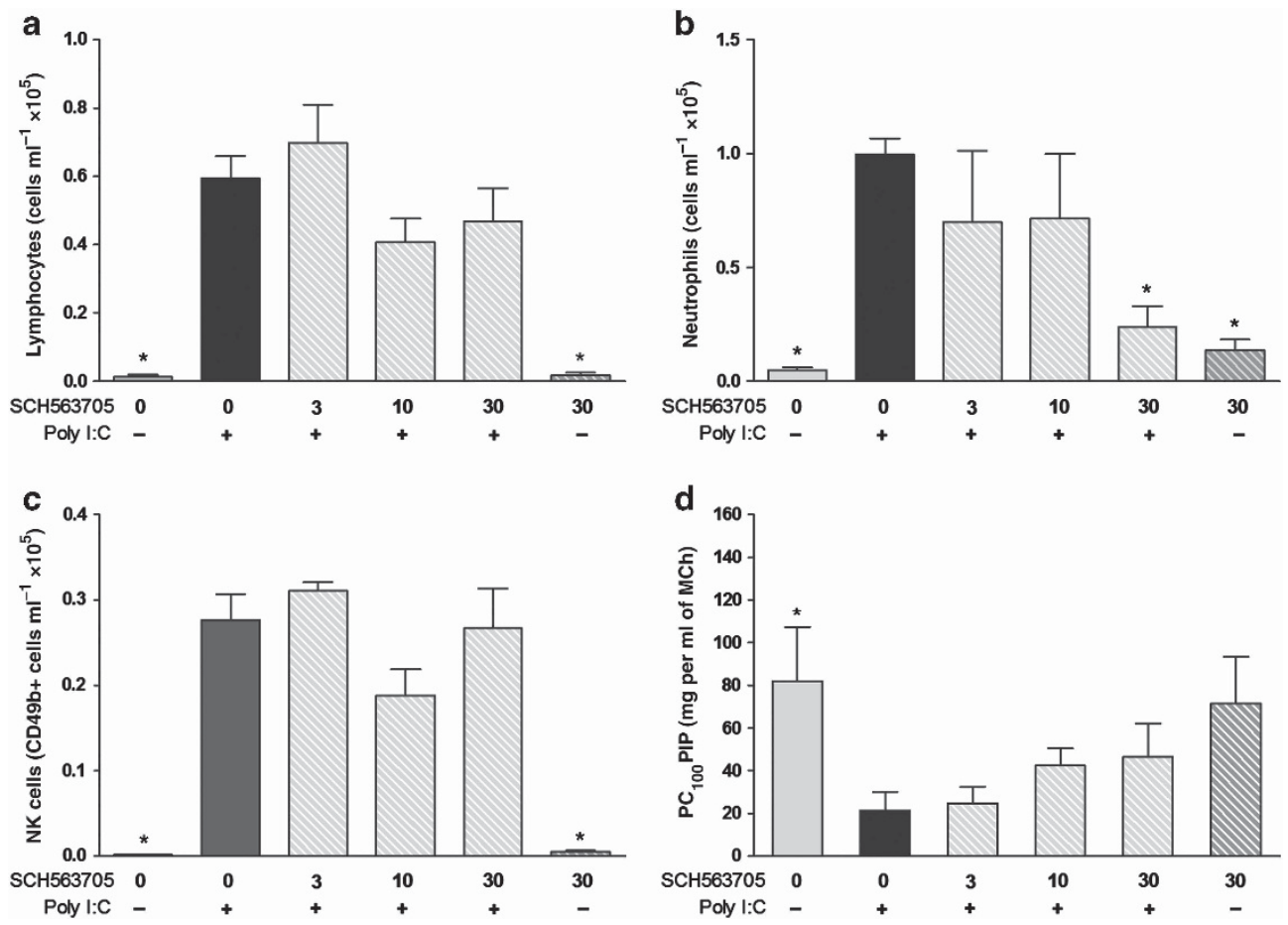

Figure 7 Effect of a CXCR2 antagonist on polyinosinic:polycytidylic acid (poly I:C)-induced inflammation. The CXCR2 antagonist, SCH563705, inhibits (b) poly I:C-induced airway neutrophil, but not (a) lymphocyte infiltrate. Neutrophil and lymphocyte numbers were determined by performing total and differential cell counts. SCH563705 also had no effect on (c) natural killer (NK) cells as determined by flow cytometric analysis or (d) airway hyper-responsiveness. Data are expressed as mean \pm S.e.m. of $n=7-8$ mice. Significance (relative to the poly I:C vehicle control) was determined using a one-way analysis of variance and is denoted as follows: ${ }^{\star} P<0.05$; ${ }^{\star \star} P<0.01$; and ${ }^{\star \star \star} P<0.001$. MCh, methacholine; $\mathrm{PC}_{100} \mathrm{PIP}$, provocative concentration producing a $100 \%$ increase in pulmonary insufflation pressure.

but did not inhibit AHR (Figure 8d). The effect on BALF cell numbers was largely due to the inhibition of lymphocyte (NK cell) infiltrate (Figure 8a,c), as well as a smaller effect on neutrophil infiltrate (Figure 8b).

\section{Clinical expression of poly I:C gene sets}

Gene sets were constructed from poly I:C-induced DEGs at individual time points. Using a novel algorithm called GSVA, ${ }^{9}$ translatability of these signatures was assessed on two publicly available clinical data sets sampling either the lungs of control subjects and COPD patients, ${ }^{14}$ or control subjects, stable IPF patients, and IPF patients with acute exacerbations ${ }^{15}$ (for a complete description of these studies, see Supplementary Methods and Supplementary Figure S6 online). The first data set from Golpon et al. ${ }^{14}$ was generated from the whole-lung tissue from "usual" emphysema ( $n=5), \alpha 1$-antitrypsin-related emphysema $(n=5)$, and controls $(n=5)$. Gene sets generated from the 2-96h poly I:C-treated mice (upregulated genes) were commonly upregulated in both emphysema patient populations, whereas these gene sets were not enriched in control samples (Figure 9a). The second data set was generated from the lungs of 23 stable IPF patients, 8 IPF patients with acute exacerbations, and 15 control subjects. Although there was enrichment of poly I:C signatures in a subset of samples, there was no distinct separation of stable or acute IPF from control samples based on up- or downregulation of poly I:C gene signatures (Figure 9b).

\section{DISCUSSION}

The inflammation elicited by dsRNA occurred over three phases starting with increased chemokine production, followed by the subsequent infiltration of neutrophils, NK cells, and finally $\mathrm{CD} 8+\mathrm{T}$ cells into the lungs. CXCR2 activation was demonstrated to be required for the neutrophil infiltrate, and CCR5 was required for the migration of the NK cells. These three distinct phases, early (2-6h), middle (24-48h), and late (72-168h), could also be defined at the molecular level by distinct gene expression signatures. GSVA revealed that these signatures are clinically relevant and specific to populations where viral exacerbations are known to contribute to disease progression (i.e., COPD patients), but not in disorders that lack this association (i.e., IPF).

Importantly, this is the first time the kinetic inflammatory changes and the molecular mechanisms driving the response to a single IN administration of poly I: $C$ have been fully described. Several groups have used poly I:C in the past to mimic the effects of a viral infection; however, most have used multiple poly I:C challenges to mimic the effect of a chronic respiratory infection. ${ }^{4,8}$ The acute response to a viral infection has been well described by many groups where increased expression of adhesion molecules and chemokines leads to the infiltration of neutrophils and NK cells, followed by CD8 + T cells and macrophages into the lungs. ${ }^{16-20}$ Here, we show that synthetic dsRNA elicits a similar response driven largely by chemo- 

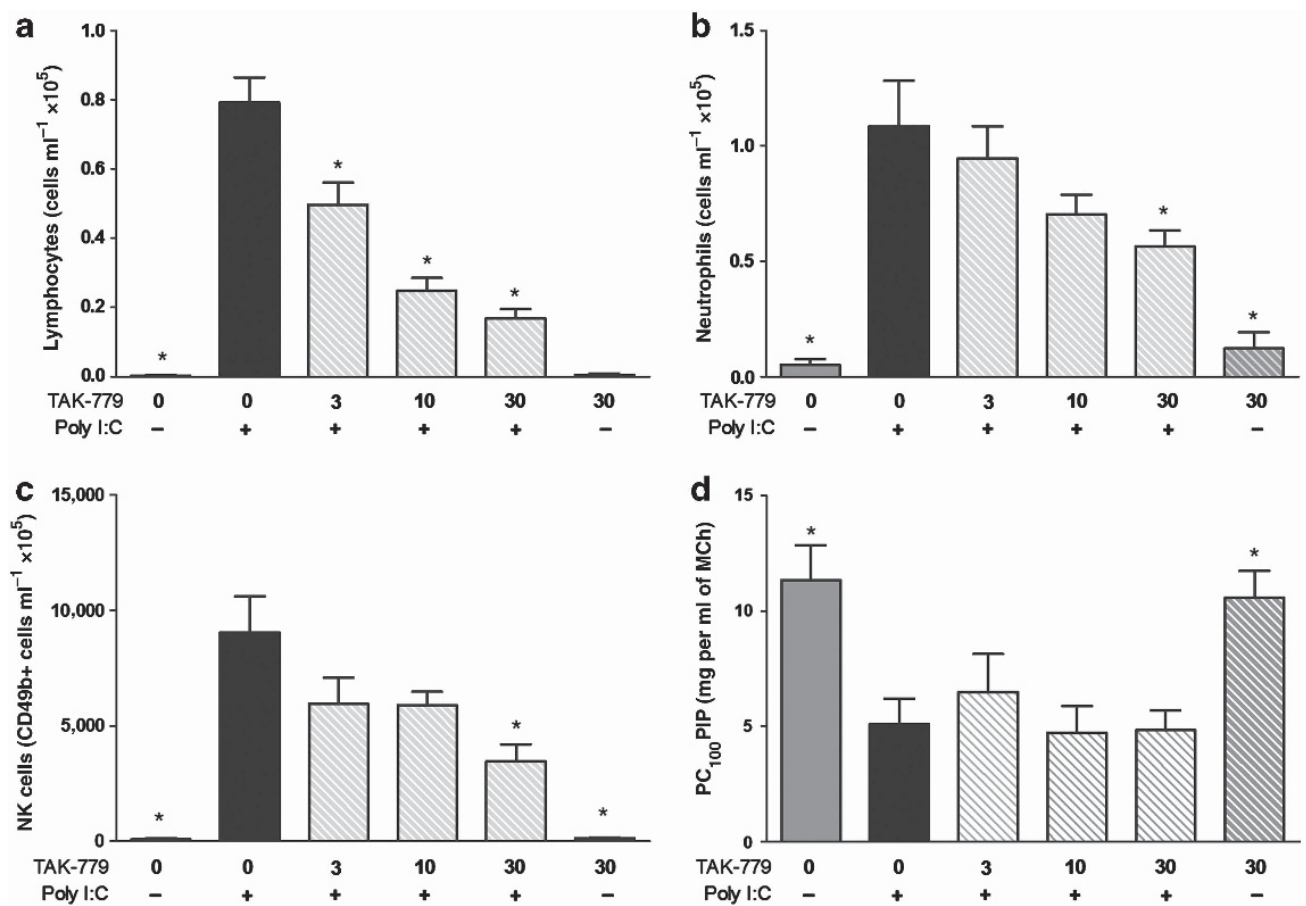

Figure 8 Effect of a CCR5 antagonist on polyinosinic:polycytidylic acid (poly l:C)-induced inflammation. The CCR5 antagonist, TAK-779, inhibits (a) poly I:C-induced airway lymphocyte but not (b) neutrophil infiltrate or (d) airway hyper-responsiveness. Neutrophil and lymphocyte numbers were determined by performing total and differential cell counts. (c) TAK-779's effect on lymphocyte numbers correlated with decreases in natural killer (NK) cells as measured by flow cytometry analysis. Data expressed as mean \pm S.e.m. of $n=9-10$. Significance (relative to the poly I:C vehicle control) was determined using a one-way analysis of variance and is denoted as follows: ${ }^{\star} P<0.05 ;{ }^{* \star} P<0.01 ;{ }^{* * \star} P<0.001$. MCh, methacholine; $P C_{100} P I P$, provocative concentration producing a $100 \%$ increase in pulmonary insufflation pressure.

a

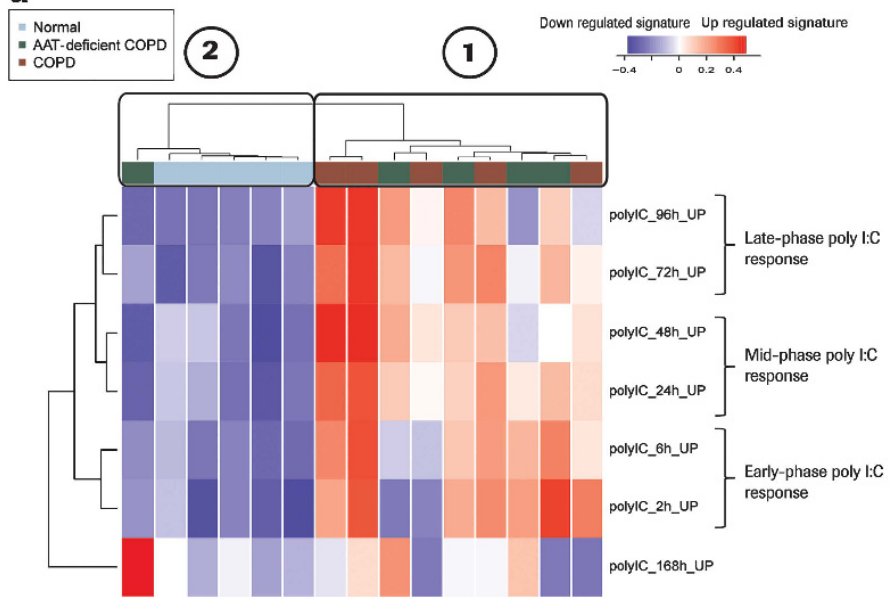

b

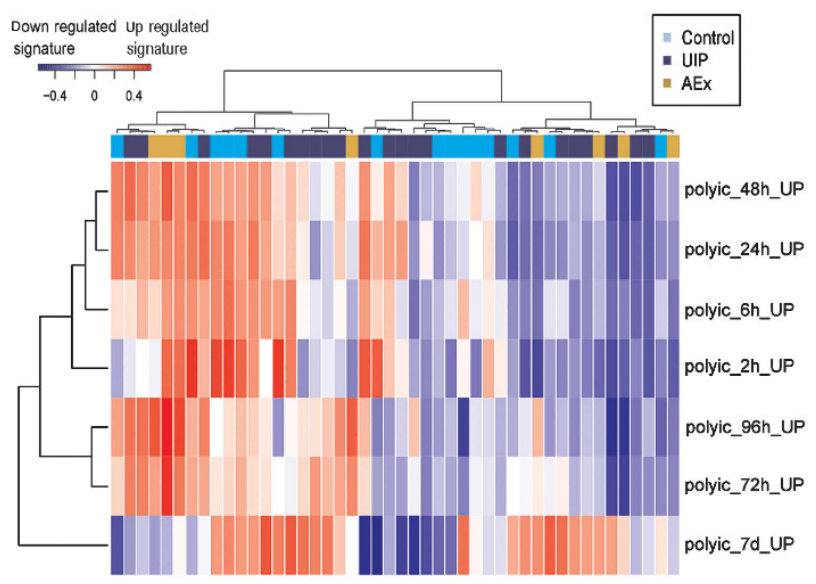

Figure 9 Gene set variation analysis identifies the enrichment of polyinosinic:polycytidylic acid (poly I:C) response gene sets in lung tissue from (a) chronic obstructive pulmonary disease (COPD) patients but not (b) idiopathic pulmonary fibrosis (IPF) patients. Columns represent lung tissue samples from human subjects from publicly available data sets (COPD: GSE1122; IPF: GSE10667), colored by disease status. Rows represent gene set variation analysis scores for individual poly I:C response gene signatures. Unsupervised hierarchical clustering of enrichment scores revealed two distinct subgroups of subjects distinguished by disease status, where cluster 1 consists primarily of COPD subjects while cluster 2 consists of mainly normal subjects (a); however, there was no discernable grouping of subjects according to IPF disease status (b). AAT, alpha 1-antitrypsin; AEx, acute exacerbator; UIP, usual interstitial pneumonia.

kines that activate the CXCR2 and CCR5 receptors, a finding also consistent with models using live virus. ${ }^{16,19,20}$ Further, at the level of gene expression many processes associated with the anti-viral response also appear to be engaged, including enrichment of TLR-activated gene sets, increased expression of type I interferons, and the expression of molecular signatures for activated monocytes, NK, and dendritic cells within the lung tissue. It is also interesting to note that the tri-phasic 
pattern of the molecular response to poly I:C exposure has been described in vitro using human myeloid and plasmacytoid dendritic cells stimulated with influenza A virus. ${ }^{21}$ Lastly, poly I:C induced AHR, which is also consistent with viral infection models; however, the mechanisms responsible for the AHR remain unknown in this system given a steroid, a CXCR2 antagonist, or a CCR5 antagonist could not affect this aspect of the model. It is therefore plausible that the AHR observed is due to mediators produced by resident lung cells.

Although mechanistically this model of poly I:C-induced airway inflammation can replicate several aspects of acute viral infections, there are shortcomings. Most notably, poly I: $\mathrm{C}$-induced airway inflammation tends to resolve more quickly compared with a live infection with a pathogen that infects mice. ${ }^{22}$ In contrast to murine pathogens (i.e., Sendai virus), it is unlikely that a single poly I:C administration will lead to chronic airway remodeling, ${ }^{23}$ although we did not evaluate the histological changes past 7 days in the current studies. It is worth pointing out, however, that models using human viruses do not appear to provide an additional benefit in terms of the clinical relevance of the system, given that the responses are exceptionally similar. ${ }^{7,24}$ This is in large part due to the fact that mice are relatively resistant to human respiratory viruses such as rhinovirus and respiratory syncytial virus unless the mice are genetically modified, the virus is adapted in vitro, or the virus is delivered at high inoculums. ${ }^{25-27}$ These are important observations given that many animal laboratories do not have the facilities or resources to allow the use of infectious pathogens within their facilities; furthermore, this model provides an alternative and the opportunity for investigators to study the effect of dsRNA exposure in isolation (single-challenge model) or in combination with other stimuli associated with chronic respiratory diseases (i.e., cigarette smoke or allergen).

Poly I:C has been used by others to exacerbate an active inflammatory response in the lungs of mice that was initially induced by cigarette smoke. ${ }^{8}$ Armed with this new information regarding the mechanisms activated by a single challenge of poly I:C, it will be interesting to understand how pathways activated by smoke and poly I:C exposure interact to produce an exacerbated response. It has been shown that NK cells from smoke-exposed mice are specifically activated and primed to hyper-respond to viral-like challenges (i.e., poly I:C, ssRNA40), although the mechanisms have not been fully elucidated. ${ }^{28}$ Additionally, cigarette smoking elicits a similar inflammatory profile to that induced by dsRNA in mice. For instance, cigarette smoke-induced inflammation is also inhibited by CXCR2 antagonism and CCR5 deletion. ${ }^{29,30}$ Further, steroids appear to have a similar effect in both the models as well, attenuating lymphocytes without affecting neutrophil infiltrates. ${ }^{31}$ Together these findings suggest that these two challenges (smoke and dsRNA) activate common pathways that can lead to additive or synergistic changes in certain mediators and subsequently an enhanced inflammatory response in the lungs.

The data presented herein are also consistent with clinical studies that have demonstrated that there are common inflammatory features associated with chronic viral infection that are observed in patients with COPD. ${ }^{32,33}$ Further supporting these findings, we provide evidence that molecular signatures induced by dsRNA are enriched in the lungs of COPD patients and can largely differentiate COPD patients from asymptomatic controls. These same signatures were not able to differentiate IPF patient populations (both stable IPF, as well as IPF patients who have recently experienced an acute exacerbation) from controls. This implies that the enrichment of these dsRNA molecular signatures is a specific feature of COPD and not a generalized feature of smoking-related lung diseases. These data are also consistent with reports that a close association exists between viral exacerbations and COPD but not IPF. In fact, clinically an acute exacerbation of IPF is only diagnosed once a viral infection is ruled out as the inciting cause. As such, these are exciting preliminary observations that a novel computational method, GSVA, was able to distinguish COPD patients from control subjects based on a molecular signature defined in a preclinical in vivo model. Previously defined gene set-enrichment methods have demonstrated differential enrichment of custom pathway signatures between different phenotypes, ${ }^{34-36}$ whereas patientspecific enrichment has only been shown using signatures defined in specific in vitro perturbation experiments. ${ }^{37,38}$ GSVA provides the added advantage of determining patient-specific enrichment scores of custom pathway signatures, which can be derived from a variety of unrelated sources. Although the use of GSVA to translate in vivo model signatures to clinical cohorts is promising, these data need to be confirmed in future studies to further enhance the link between poly I:C signatures and COPD cohorts. Specifically, prospective studies using better defined cohorts, which include viral exacerbation, comprehensive lung function, and smoking history data are required to effectively validate this association.

In conclusion, these data suggest that poly I:C can be used to efficiently model aspects of the host response to an acute respiratory viral infection. Our data demonstrate that CXCR2 and CCR5 inhibitors attenuate different components of the response to dsRNA. Efforts to develop specific chemokine receptor antagonists have failed to achieve clinical efficacy owing to immune system redundancy. Therefore, a dual antagonist approach that attenuates different components of the immune system to impact disease progression may be more efficacious. Using novel molecular profiling methods, we translated the observations made in this preclinical model to clinical samples from COPD but not IPF patients. These data imply that a dual CXCR2/CCR5 antagonist may be an effective therapeutic approach for a subset of COPD patients.

\section{METHODS}

Animals. Male BALB/cJ mice (7-9-weeks old) were purchased from the Jackson Laboratory (Bar Harbor, ME), housed under pathogen-free conditions with food and water ad libitum. All animal care and experimental procedures were approved by the Roche Animal Care and Use Committee, which is a facility accredited by the American Association for the Accreditation of Laboratory Animal Care (AAALAC).

Administration of poly I:C. Mice were anesthetized with isoflurane and IN administered saline or high-molecular weight poly I:C 
(InvivoGen, San Diego, CA) at different doses (3-300 $\mu$ g per animal) in $50 \mu \mathrm{l}$ sterile saline. Poly I:C was freshly prepared for each experiment according to the manufacturer's protocol. For time-course studies, a sub-maximal dose of $30 \mu \mathrm{g}$ was administered IN once and assessed from 2 to $168 \mathrm{~h}$, as mentioned in the figure legends.

Lung function. Non-invasive AHR (Penh) measurements are described in more detail in the Supplementary Method section online. Invasive lung function measurements were taken $24 \mathrm{~h}$ following poly I:C administration. Mice were anesthetized with $2.5 \mathrm{mg}$ sodium pentobarbital (Abbott Labs, IL), and the trachea cannulated. Animals were mechanically ventilated using a computer-controlled piston ventilator (flexiVent, SCIREQ, Montreal, Quebec, Canada; tidal volume $=10 \mathrm{ml} \mathrm{kg}^{-1}$, respiratory rate of 150 breaths $\mathrm{min}^{-1}, 3 \mathrm{~cm} \mathrm{H}_{2} \mathrm{O}$ positive end-expiratory pressure). Baseline PIP was measured by a pressure transducer and data were digitally recorded using flexiWare 7 software (SCIREQ, Montreal, Quebec, Canada). Bronchoconstriction was measured as an increase in $\mathrm{PIP}^{39}$ that was elicited after 10 inspirations of saline aerosol generated by an inline ultrasonic nebulizer. Mice were challenged with rising halflogarithmic doses of methacholine beginning at $3 \mathrm{mg} \mathrm{ml}^{-1}$ and ending when baseline PIP had doubled. AHR was determined by a decrease in the provocative concentration of nebulized methacholine needed to induce a $100 \%$ increase in the baseline PIP.

Lung inflammation. Immediately following lung function measurements, mice were exsanguinated and blood collected for analyte or compound level determinations. To perform bronchoalveolar lavage (BAL), the lungs were washed with $4 \times 0.3 \mathrm{ml}$ phosphate-buffered saline. After lavage, lungs were snap frozen in liquid nitrogen for subsequent RNA isolation and hybridization to Affymetrix gene expression arrays (Affymetrix Inc., Santa Clara, CA) (see below). Total leukocyte numbers were determined on a Z1 coulter counter (Beckman Coulter, Fullerton, CA). For differential leukocyte counts, cytospin preparations were stained with Diff-Quik (DADE Diagnostics, West Monroe, CA) and 200 cells were counted using the standard morphological criteria. Remaining BAL fluid supernatants were stored at $-80^{\circ} \mathrm{C}$ until analyzed for cytokine and chemokine levels. Cell pellets were resuspended in phosphate-buffered saline for further phenotyping using flow cytometry as described below.

Flow cytometry. Multi-parameter (up to six color) analyses of stained BALF single-cell suspensions were performed on an LSR II (BD Biosciences, San Jose, CA) and analyzed using Flow Jo software (TreeStar, Ashland, OR). Briefly, cells were pre-incubated with the BD Fc block solution (purified rat IgG2a anti-mouse CD16/CD32 monoclonal antibody). Cells were then stained with fluorochrome-conjugated monoclonal antibodies obtained from $\mathrm{BD}$, washed, and resuspended in $\mathrm{BD}$ stabilizing fixative before analysis. The following purified mouse antibodies were used for staining: CD45-FITC (clone 30-F11), CD3-PerCp/Cy5.5 (clone 145-2C11), CD4-V450 (clone L3T4), CD8-Alexa700 (clone 53-6.7), and CD49b-APC (clone DX5). Analysis was based on data collected from at least 100,000 events. Unless otherwise stated, two samples within respective groups were combined and analyzed together. The sample sizes reported within each figure legend correspond to the total number of mice used to generate the data.

Histology. Mice were terminated with an overdose of sodium pentobarbital and the trachea cannulated. Lungs were infused at a pressure of $25 \mathrm{~cm}$ of $\mathrm{H}_{2} \mathrm{O}$ with $1.0 \%$ Type IX agarose $\left(40^{\circ} \mathrm{C}\right.$ at the time of infusion). After infusion, the trachea was ligated and the lungs removed intact and chilled in ice-cold phosphate-buffered saline. After the infused agar had solidified (approximately $10 \mathrm{~min}$ ), the lungs were separated. The left lobe was placed in a labeled tissue cassette and fixed in $10 \%$ neutral buffered saline, processed, and routinely embedded in paraffin. The blocks were sectioned at a thickness of 5- $\mu \mathrm{m}$ longitudinally at the level of bifurcation of the major bronchus and the sections stained with hematoxylin and eosin and alcian blue $(\mathrm{AB}) /$ periodic acid-Schiff for examination.
Cytokine and chemokine measurements. Mouse cytokine and chemokine levels in the BAL fluid were measured using MSD multiplex kits (Gaithersburg, MD) and Millipore immunoassay kits (Billerica, MA). The assays were performed following the manufacturer's protocol. The levels of TNF- $\alpha$, GM-CSF (granulocyte macrophages colony-stimulating factor), KC, Eotaxin, RANTES, IFN- $\gamma$, TARC, CCL21, IL-1 $\beta$, IL-6, IL-2, IL-4, IL-5, IL-10, IL-12 IL-13, IL-16, and IL-17 were measured. The limit of detection ranged from 0.9 to $10 \mathrm{pg} \mathrm{ml}^{-1}$.

Small molecule efficacy studies. Budesonide $\left(0.3-10 \mathrm{mg} \mathrm{kg}^{-1}\right)$ (SigmaAldrich, St Louis, MO) or its vehicle ( $2 \%$ Klucel, $0.1 \%$ Tween 80 in water) were administered per os (p.o.) $1 \mathrm{~h}$ before poly I:C administration. The CXCR2 antagonist, Sch563705 (3-30 $\left.\mathrm{mg} \mathrm{kg}^{-1}\right)$ or its vehicle (2\% Klucel, $0.1 \%$ Tween 80 in $\mathrm{H}_{2} \mathrm{O}$ ) were administered p.o. $1 \mathrm{~h}$ before and $6 \mathrm{~h}$ after poly I:C challenge. The CCR5 antagonist, TAK-779, or the vehicle (saline) were administered p.o. $1 \mathrm{~h}$ before and $6 \mathrm{~h}$ after poly I:C challenge. Volumes dosed for both compounds were on a $10 \mathrm{ml} \mathrm{kg}^{-1}$ basis. Lung function and BALF inflammation were assessed $24 \mathrm{~h}$ later.

Microarray processing and data analysis. Total RNA was isolated from the mouse lung tissue of poly I:C and saline-treated mice across seven time points $(2,6,24,48,72,96$, and $168 \mathrm{~h}, n=6$ per group $)$ and homogenized in QIAzol reagent. Purified total RNA was amplified and labeled using NuGen Ovation kits (NuGEN Technologies, San Carlos, CA) and RNA from samples was hybridized to Affymetrix Mouse 4302.0 arrays. Following microarray data preprocessing, murine genes were mapped to their human orthologs for subsequent pathway analysis. This yielded 14,300 unique mouse genes that mapped to human orthologs and were subsequently used for analysis. DEGs were determined using an analysis of variance, with pairwise comparisons between poly I:C and saline treatment at each time point. $P$ values for DEGs in pairwise comparisons were adjusted using a Benjamini-Hochberg correction to account for multiple hypothesis testing. ${ }^{40}$ Genes that were significantly altered at least twofold between poly I:C treatment and saline controls $(F D R<0.05)$ were considered to be differentially expressed. Unsupervised hierarchical clustering was performed on the union of DEGs between poly I:C and saline-treated samples across all time points to determine phases of response to poly I:C treatment. Common and unique genes between these phases of response were determined for each phase by taking the union and intersection of DEGs.

Gene signatures of interest were evaluated in the murine poly I:C expression data set using GSEA ${ }^{36}$ to determine altered expression of signaling pathways in response to poly I:C treatment at each time point. Briefly, enrichment of gene sets was calculated against the entire set of 14,300 genes from the poly I:C treatment data set, ranked based on a composite score of fold-change and FDR differences between saline and poly I:C treatment at each time point. FDR values were determined for enrichment of gene sets by permuting gene sets.

Modular analysis of previously defined blood transcriptome gene modules was performed as described by Chaussabel et al. ${ }^{11}$ to determine enrichment of these modules among DEGs from poly I:C-treated samples across time points. The degree of overlap and the directionality of poly I:C-induced genes at each time point were determined for each transcriptome module.

Enrichment of poly I:C genes in clinical datasets was determined using the GSVA algorithm, ${ }^{9}$ as implemented in the R software environment (http://www.r-project.org/). For more details on array data preprocessing, gene ontology, GSEA, modular analysis, and GSVA, see Supplementary Methods online.

Additional statistical analysis. Data are expressed as mean \pm s.e.m. unless specified otherwise. Unless stated otherwise elsewhere in the methods, statistical analyses of time course studies were determined using a Student's $t$-test, while compound efficacy studies were determined using a one-way analysis of variance and differences identified using the 
Dunnett's multiple comparison test using GraphPad Prism (version 5.03, GraphPad Software Inc., La Jolla, CA).

SUPPLEMENTARY MATERIAL is linked to the online version of the paper at http://www.nature.com/mi

\section{ACKNOWLEDGMENTS}

We thank Lorena Renteria and Jacob LaStant for their assistance with the preparation and harvest of the lung tissues, and Justin Guinney for his input on the application of GSVA to this data set.

\section{DISCLOSURE}

The authors declared no conflict of interest.

() 2013 Society for Mucosal Immunology

\section{REFERENCES}

1. Mohan, A. et al. Prevalence of viral infection detected by PCR and RT-PCR in patients with acute exacerbation of COPD: a systematic review. Respirology 15, 536-542 (2010).

2. Rohde, G. et al. Respiratory viruses in exacerbations of chronic obstructive pulmonary disease requiring hospitalisation: a case-control study. Thorax 58, 37-42 (2003).

3. Seemungal, T. et al. Respiratory viruses, symptoms, and inflammatory markers in acute exacerbations and stable chronic obstructive pulmonary disease. Am. J. Respir. Crit. Care Med. 164, 1618-1623 (2001).

4. Stowell, N.C. et al. Long-term activation of TLR3 by poly(l:C) induces inflammation and impairs lung function in mice. Respir. Res. 10, 43 (2009).

5. Groskreutz, D.J., Monick, M.M., Powers, L.S., Yarovinsky, T.O., Look, D.C. \& Hunninghake, G.W. Respiratory syncytial virus induces TLR3 protein and protein kinase $R$, leading to increased double-stranded RNA responsiveness in airway epithelial cells. J. Immunol. 176, 1733-1740 (2006).

6. Le Goffic, R. et al. Cutting edge: influenza A virus activates TLR3dependent inflammatory and RIG-I-dependent antiviral responses in human lung epithelial cells. J. Immunol. 178, 3368-3372 (2007).

7. Wang, Q. et al. MDA5 and TLR3 initiate pro-inflammatory signaling pathways leading to rhinovirus-induced airways inflammation and hyperresponsiveness. PLoS Pathogens 7, e1002070 (2011).

8. Kang, M.J. et al. Cigarette smoke selectively enhances viral PAMP- and virus-induced pulmonary innate immune and remodeling responses in mice. J. Clin. Invest. 118, 2771-2784 (2008).

9. GSVA-The Gene Set Variation Analysis Package. http://www. bioconductor.org/packages/2.8/bioc/html/GSVA.html2011, Accessed Date Accessed 2011 Accessed.

10. Abbas, A.R., Wolslegel, K., Seshasayee, D., Modrusan, Z. \& Clark, H.F. Deconvolution of blood microarray data identifies cellular activation patterns in systemic lupus erythematosus. PloS One 4, e6098 (2009).

11. Chaussabel, D. et al. A modular analysis framework for blood genomics studies: application to systemic lupus erythematosus. Immunity 29, 150-164 (2008).

12. Chao, J. et al. C(4)-alkyl substituted furanyl cyclobutenediones as potent, orally bioavailable CXCR2 and CXCR1 receptor antagonists. Bioorg. Med. Chem. Lett. 17, 3778-3783 (2007).

13. Baba, M. et al. A small-molecule, nonpeptide CCR5 antagonist with highly potent and selective anti-HIV-1 activity. Proc. Natl. Acad. Sci. USA 96, 5698-5703 (1999).

14. Golpon, H.A. et al. Emphysema lung tissue gene expression profiling. Am. J. Respir. Cell Mol. Biol. 31, 595-600 (2004).

15. Konishi, K. et al. Gene expression profiles of acute exacerbations of idiopathic pulmonary fibrosis. Am. J. Respir. Crit. Care Med. 180, 167-175 (2009).

16. Kohlmeier, J.E. et al. The chemokine receptor CCR5 plays a key role in the early memory CD8+ T cell response to respiratory virus infections. Immunity 29, 101-113 (2008)

17. Stein-Streilein, J., Bennett, M., Mann, D. \& Kumar, V. Natural killer cells in mouse lung: surface phenotype, target preference, and response to local influenza virus infection. J. Immunol. 131, 2699-2704 (1983).

18. Verbist, K.C., Field, M.B. \& Klonowski, K.D. Cutting edge: IL-15 independent maintenance of mucosally generated memory CD8 T cells. J. Immunol. 186, 6667-6671 (2011).
19. Wareing, M.D., Shea, A.L., Inglis, C.A., Dias, P.B. \& Sarawar, S.R. CXCR2 is required for neutrophil recruitment to the lung during influenza virus infection, but is not essential for viral clearance. Viral. Immunol. 20, 369-378 (2007).

20. Weiss, G., Christensen, H.R., Zeuthen, L.H., Vogensen, F.K., Jakobsen, M. \& Frokiaer, H. Lactobacilli and bifidobacteria induce differential interferonbeta profiles in dendritic cells. Cytokine 56, 520-530 (2011).

21. Piqueras, B., Connolly, J., Freitas, H., Palucka, A.K. \& Banchereau, J. Upon viral exposure, myeloid and plasmacytoid dendritic cells produce 3 waves of distinct chemokines to recruit immune effectors. Blood 107, 2613-2618 (2006)

22. Tyner, J.W. et al. CCL5-CCR5 interaction provides antiapoptotic signals for macrophage survival during viral infection. Nat. Med. 11, 1180-1187 (2005).

23. Kim, E.Y. et al. Persistent activation of an innate immune response translates respiratory viral infection into chronic lung disease. Nat. Med. 14, 633-640 (2008).

24. Nagarkar, D.R. et al. CXCR2 is required for neutrophilic airway inflammation and hyperresponsiveness in a mouse model of human rhinovirus infection. J. Immunol. 183, 6698-6707 (2009).

25. Bartlett, N.W. et al. Mouse models of rhinovirus-induced disease and exacerbation of allergic airway inflammation. Nat. Med. 14, 199-204 (2008).

26. Graham, B.S., Perkins, M.D., Wright, P.F. \& Karzon, D.T. Primary respiratory syncytial virus infection in mice. J. Med. Virol. 26, 153-162 (1988).

27. Holtzman, M.J. et al. Immune pathways for translating viral infection into chronic airway disease. Adv. Immunol. 102, 245-276 (2009).

28. Motz, G.T. et al. Chronic cigarette smoke exposure primes NK cell activation in a mouse model of chronic obstructive pulmonary disease. J. Immunol. 184, 4460-4469 (2010).

29. Ma, B. et al. Role of CCR5 in IFN-gamma-induced and cigarette smoke-induced emphysema. J. Clin. Invest. 115, 3460-3472 (2005).

30. Stevenson, CS et al. Characterization of cigarette smoke-induced inflammatory and mucus hypersecretory changes in rat lung and the role of CXCR2 ligands in mediating this effect. Am. J. Physiol. Lung Cell. Mol. Physiol. 288, L514-L522 (2005).

31. Wan, W.Y. et al. Pharmacological characterisation of anti-inflammatory compounds in acute and chronic mouse models of cigarette smokeinduced inflammation. Respir. Res. 11, 126 (2010).

32. Saetta, M. et al. CD8+ve cells in the lungs of smokers with chronic obstructive pulmonary disease. Am. J. Respir. Crit. Care Med. 160, 711-717 (1999).

33. Sikkel, M.B., Quint, J.K., Mallia, P., Wedzicha, J.A. \& Johnston, S.L. Respiratory syncytial virus persistence in chronic obstructive pulmonary disease. Pediatr. Infect. Dis. J. 27 (10 Suppl), S63-S70 (2008).

34. Barry, W.T., Nobel, A.B. \& Wright, F.A. Significance analysis of functional categories in gene expression studies: a structured permutation approach. Bioinformatics 21, 1943-1949 (2005).

35. Pavlidis, P., Qin, J., Arango, V., Mann, J.J. \& Sibille, E. Using the gene ontology for microarray data mining: a comparison of methods and application to age effects in human prefrontal cortex. Neurochem. Res. 29, 1213-1222 (2004).

36. Subramanian, A. et al. Gene set enrichment analysis: a knowledge-based approach for interpreting genome-wide expression profiles. Proc. Natl. Acad. Sci. USA 102, 15545-15550 (2005).

37. Bild, A.H. et al. Oncogenic pathway signatures in human cancers as a guide to targeted therapies. Nature 439, 353-357 (2006).

38. Gustafson, A.M. et al. Airway PI3K pathway activation is an early and reversible event in lung cancer development. Sci. Translational Med. 2, 26ra25 (2010).

39. Konzett, H.R. Versuchsanordnung zu Untersuchungen an der Bronchialmuskulatur. Naunyn-Schmiedeberg's Arch. Pharmacol 195, 71-74 (1940)

40. Benjamini, Y. \& Hochberg, Y. Controlling the false discovery rate-a practical and powerful approach to multiple testing. J. R. Stat. Soc. B Met. 57, 289-300 (1995).

\section{(c)} SOWERIEHISRESERVED

This work is licensed under the Creative Commons Attribution-NonCommercial-No Derivative Works 3.0

Unported License. To view a copy of this license, visit http://creativecommons.org/licenses/by-nc-nd/3.0/ 